\title{
Intravenous immunoglobulin mediates anti-inflammatory effects in peripheral blood mononuclear cells by inducing autophagy
}

\author{
Mrinmoy Das', Anupama Karnam, Emmanuel Stephen-Victor', Laurent Gilardin ${ }^{1}{ }^{1,2}$, Bharat Bhatt ${ }^{3}$, \\ Varun Kumar Sharma', Naresh Rambabu', Veerupaxagouda Patil ${ }^{1}$, Maxime Lecerf ${ }^{1,4}$, Fabian Käsermann ${ }^{5}$, \\ Patrick Bruneval ${ }^{6}$, Kithiganahalli Narayanaswamy Balaji ${ }^{3}$, Olivier Benveniste ${ }^{2,7}$, Srini V. Kaveri ${ }^{1,4}$ and Jagadeesh Bayry (1) ${ }^{1,4}$
}

\begin{abstract}
Autophagy plays an important role in the regulation of autoimmune and autoinflammatory responses of the immune cells. Defective autophagy process is associated with various autoimmune and inflammatory diseases. Moreover, in many of these diseases, the therapeutic use of normal immunoglobulin $G$ or intravenous immunoglobulin (IVIG), a pooled normal IgG preparation, is well documented. Therefore, we explored if IVIG immunotherapy exerts therapeutic benefits via induction of autophagy in the immune cells. Here we show that IVIG induces autophagy in peripheral blood mononuclear cells (PBMCs). Further dissection of this process revealed that IVIG-induced autophagy is restricted to inflammatory cells like monocytes, dendritic cells, and M1 macrophages but not in cells associated with Th2 immune response like M2 macrophages. IVIG induces autophagy by activating AMP-dependent protein kinase, beclin1, class III phosphoinositide 3-kinase and p38 mitogen-activated protein kinase and by inhibiting mammalian target of rapamycin. Mechanistically, IVIG-induced autophagy is $F\left(a b^{\prime}\right)_{2}$-dependent but sialylation independent, and requires endocytosis of IgG by innate cells. Inhibition of autophagy compromised the ability of IVIG to suppress the inflammatory cytokines in innate immune cells. Moreover, IVIG therapy in inflammatory myopathies such as dermatomyositis, antisynthetase syndrome and immune-mediated necrotizing myopathy induced autophagy in PBMCs and reduced inflammatory cytokines in the circulation, thus validating the translational importance of these results. Our data provide insight on how circulating normal immunoglobulins maintain immune homeostasis and explain in part the mechanism by which IVIG therapy benefits patients with autoimmune and inflammatory diseases.
\end{abstract}

\section{Background}

Recent reports show that macroautophagy, hereafter referred to as autophagy, plays an important role in the regulation of autoimmune and autoinflammatory responses. Autophagy involves lysosomal degradation of unnecessary or dysfunctional cellular components,

\footnotetext{
Correspondence: Jagadeesh Bayry (jagadeesh.bayry@crc.jussieu.fr) ${ }^{1}$ Institut National de la Santé et de la Recherche Médicale; Centre de Recherche des Cordeliers, Equipe- Immunopathologie et Immunointervention Thérapeutique, Sorbonne Université, 75006 Paris, France

2Département de Médecine Interne et Immunologie Clinique, Hôpital PitiéSalpêtrière, AP-HP, 75013 Paris, France

Full list of author information is available at the end of the article

Edited by G. M. Fimia
}

cytotoxic mediators and misfolded proteins. During this process, targeted cytoplasmic constituents are isolated from the rest of the cell within a double-membraned vesicle known as an autophagosome. The autophagosome then fuses with a lysosome and its cargo is degraded and recycled $^{1,2}$. A series of autophagy-related proteins (ATG) and several kinases play an important role in the regulation of autophagy. While mammalian target of rapamycin (mTOR) and AKT negatively regulate autophagy process, kinases such as phosphoinositide 3-kinase (PI3K) class III, AMP-dependent protein kinase (AMPK), extracellular signal-regulated kinase (ERK) and Jun $\mathrm{N}$-terminal kinase (JNK) positively regulate components of autophagy

\section{(c) The Author(s) 2020}

(c) (i) Open Access This article is licensed under a Creative Commons Attribution 4.0 International License, which permits use, sharing, adaptation, distribution and reproduction cc) in any medium or format, as long as you give appropriate credit to the original author(s) and the source, provide a link to the Creative Commons license, and indicate if changes were made. The images or other third party material in this article are included in the article's Creative Commons license, unless indicated otherwise in a credit line to the material. If material is not included in the article's Creative Commons license and your intended use is not permitted by statutory regulation or exceeds the permitted use, you will need to obtain permission directly from the copyright holder. To view a copy of this license, visit http://creativecommons.org/licenses/by/4.0/. 
machinery. p38 mitogen-activated protein kinase (p38 MAPK) has a dual role in regulating autophagy ${ }^{3}$.

Autophagy was initially investigated as a process involved in the "cell survival" and nowadays is recognized as fundamental in the physiology of eukaryotic cells. Autophagy also plays a fundamental role in the regulation of innate and adaptive immune responses ${ }^{4}$, lymphocyte differentiation, survival and homeostasis ${ }^{4,5}$. Several reports show the role of autophagy in the regulation of autoimmune and inflammatory pathologies including systemic lupus erythematosus, inflammatory bowel diseases, rheumatoid arthritis, psoriasis, multiple sclerosis and myositis ${ }^{6-15}$ and hence autophagy represents potential target to treat autoimmune and inflammatory diseases $^{16}$. Moreover, in many of these diseases, the therapeutic use of intravenous and subcutaneous immunoglobulin (IVIG/SCIG), the normal human IgG preparations obtained from the pools of plasma of several thousand healthy donors is well documented ${ }^{17-20}$.

In addition to its use in primary and secondary immune deficiencies, IVIG is increasingly used for the treatment of large number of autoimmune and inflammatory diseases $^{17-20}$. Data from various labs have demonstrated that IVIG exerts immune homeostasis by suppressing the activation of innate and adaptive immune compartments, inhibiting the inflammatory mediators and enhancing the anti-inflammatory processes ${ }^{21-24}$. In view of common important role played by autophagy and IVIG in the regulation of autoimmune and inflammatory responses, we aimed to explore if IVIG immunotherapy exerts immune homeostasis and therapeutic benefits via induction of autophagy.

\section{Results}

IVIG induces autophagy in peripheral blood mononuclear cells of healthy donors

In order to investigate whether IVIG induces autophagy, we first resorted to peripheral blood mononuclear cells (PBMCs) of healthy donors. PBMCs were cultured with different doses of IVIG (10 and $25 \mathrm{mg} / \mathrm{ml}$ ) for $24 \mathrm{~h}$ and analyzed by western blot for the level of LC3-II, one of the well-characterized markers of autophagy ${ }^{25}$. Untreated PBMCs showed minimal level of LC3-II and its expression was not altered by equimolar concentrations of human serum albumin (HSA), an irrelevant protein control for IVIG. However, treatment of PBMCs with IVIG led to significantly enhanced level of LC3-II (Fig. 1a). Further, the effect of IVIG on autophagy was dose-dependent. High level of LC3-II was observed at $25 \mathrm{mg} / \mathrm{ml}$, a concentration of IVIG reaches in the circulation of autoimmune patients immediately following therapy. As LC3II was also enhanced in cells treated with $10 \mathrm{mg}$

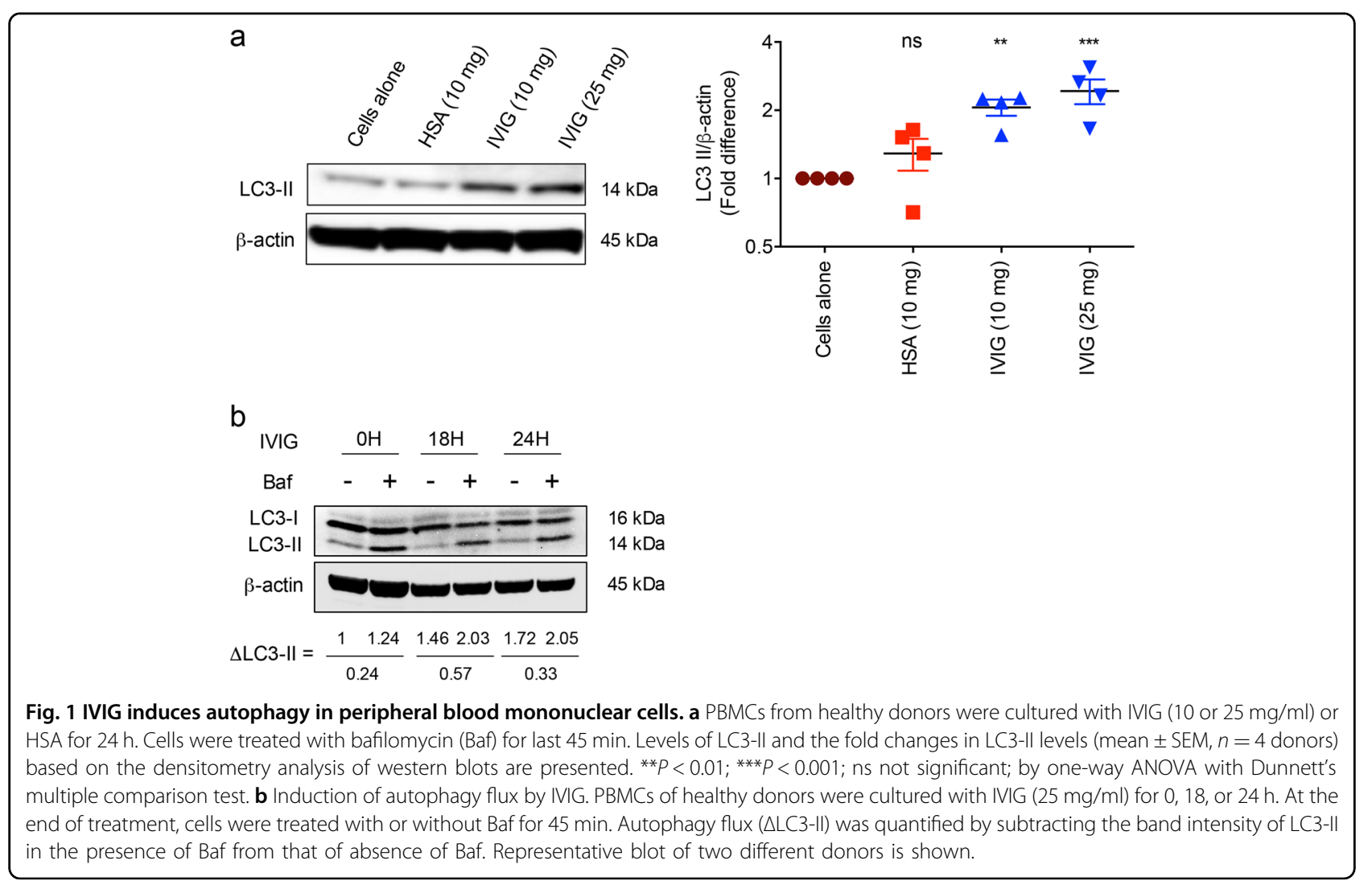


concentration of IVIG, which correspond to IgG in the circulation of healthy individuals, this suggests that circulating normal IgG induces autophagy under physiological conditions. Induction of autophagy by IVIG was also confirmed through the analyses of autophagy flux (Fig. 1b).

IVIG induces autophagy in monocytes, dendritic cells, and M1 macrophages, but not in M2 macrophages

As innate immune cells are central for initiating and propagating the immune responses, we then explored if the ability of IVIG to induce autophagy is universal for all types of innate immune cells. To address this question, we exposed peripheral blood monocytes, and monocytederived dendritic cells (DCs), M1 macrophages and M2 macrophages to different doses of IVIG (10 and $25 \mathrm{mg} /$ $\mathrm{ml})$. We found that among innate immune cells, IVIGinduced autophagy was restricted to inflammatory cells like monocytes, DCs and M1 macrophages but not in cells associated with Th2 immune response like M2 macrophages (Fig. 2a-d). We further confirm that inability of IVIG to induce autophagy in M2 macrophages is not due to technical errors or defects in the cells to undergo autophagy as starvation condition could induce autophagy in M2 cells (Fig. 2d). These results thus suggest that IVIG exhibits selective ability to induce autophagy and that all immune cells are not susceptible to IVIG-induced autophagy.

In order to obtain additional evidence on the IVIGinduced autophagy, we performed transmission electron microscopy of lipopolysaccharide-stimulated DCs to visualize autophagic organelles. While control DCs did not show autophagosome structures in the cytoplasm, IVIG-treated DCs on the other hand displayed doublemembraned autophagosomes with undigested or partially degraded cytoplasmic contents (including endoplasmic reticulum) (Fig. 3a, b). These data thus validate that IVIG induces autophagy in inflammatory immune cells.

IVIG-induced autophagy is associated with induction and activation of AMPK, beclin-1, class III PI3K and P38MAPK, and inhibition of mTOR activation

Several kinases and signaling molecules play an important role in the regulation of autophagy ${ }^{1,3}$. Therefore, to gain further insight on the signaling molecules implicated in IVIG-induced autophagy, we probed the expression and activation status of various signaling molecules that are critical for the formation of autophagosome. All experiments for the dissection of mechanisms were performed in monocytes. We found that IVIG significantly reduced the phosphorylated form of mTOR without modulating total mTOR expression (Fig. 4a, b). On the other hand, IVIG induced beclin-1 and class III PI3K and significantly enhanced the phosphorylation of p38 MAPK and AMPK (Fig. 4a, c-f).
Inhibition of Class III PI3K, AMPK and p38 MAPK activation leads to abrogation of IVIG-induced autophagy

To validate the role of Class III PI3K, AMPK and p38 MAPK kinases in IVIG-induced autophagy, monocytes were pretreated with different pharmaceutical inhibitors, i.e., 3-methyladenine (3MA, for class III PI3K), SB202190 (for p38 MAPK) and compound C (for AMPK) followed by culture with IVIG for $24 \mathrm{~h}$. In line with the data on activation of these kinases by IVIG (Fig. 4), inhibition of Class III PI3K, AMPK and p38 MAPK kinases led to the abrogation of IVIG-induced autophagy as analyzed by LC3-II protein level by western blot (Fig. 5a-c).

To further authenticate IVIG-induced autophagy, we resorted to fluorescence microscopy to visualize LC3 puncta in IVIG-treated THP-1 cells, a human monocytic cell line. We found significantly higher characteristic LC3 puncta formation in the cells upon treatment with IVIG (Fig. 5d, e), thus confirming the western blot results. Moreover, pretreatment of THP-1 cells with 3MA, SB202190 and compound C significantly abolished IVIGinduced LC3 puncta formation (Fig. 5d, e). These results thus demonstrate that IVIG induces autophagy through activation of class III PI3K, p38 MAPK and AMPK kinases.

\section{Induction of autophagy by IVIG is $F\left(a b^{\prime}\right)_{2}$-dependent but sialylation independent}

IgG consists of $\mathrm{Fc}$ and Fab regions. While some mechanisms of IVIG were dependent on Fc-fragments, other mechanisms were mediated via $\mathrm{F}\left(\mathrm{ab}^{\prime}\right)_{2}$ fragments $^{21}$. Therefore, we investigated the role of $\mathrm{F}\left(\mathrm{ab}^{\prime}\right)_{2}$ fragments to dissect the mechanisms of IVIG-induced autophagy. Monocytes were cultured with equimolar concentrations of either IVIG or $\mathrm{F}\left(\mathrm{ab}^{\prime}\right)_{2}$ fragments for $24 \mathrm{~h}$. Interestingly, $\mathrm{F}\left(\mathrm{ab}^{\prime}\right)_{2}$ fragments of IVIG significantly enhanced the levels of LC3-II (Fig. 6a). These results thus demonstrate that autophagy induction by IVIG is $\mathrm{F}\left(\mathrm{ab}^{\prime}\right)_{2}$-dependent.

Sialylation of IgG has been proposed to mediate several anti-inflammatory actions of IVIG though other reports also demonstrate sialylation-independent anti-inflammatory effects ${ }^{26-33}$. Therefore, we investigated if IVIGinduced autophagy is dependent on its sialylation content. However, we found that desialylation of IgG had no repercussion on IVIG-induced autophagy (Fig. 6b).

\section{Endocytosis of IgG is mandatory for the IVIG-mediated autophagy in innate immune cells}

Numerous reports have shown that innate immune cells internalize IVIG by endocytosis ${ }^{28,34,35}$. We therefore investigated whether signaling cascades induced by IVIG upon binding of IgG to the surface of innate immune cells is sufficient for inducing autophagy or it requires endocytosis of IgG. To probe this hypothesis, monocytes were pretreated with cytochalasin D, an inhibitor of endocytosis followed by culture with IVIG for $24 \mathrm{~h}$. As shown in 

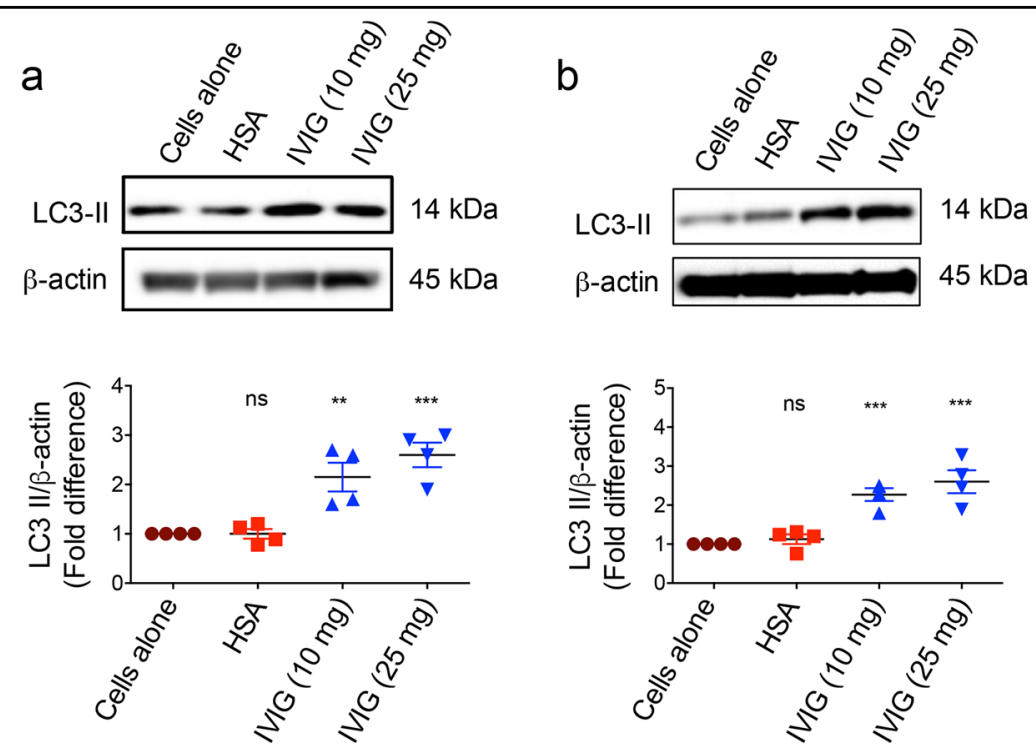

Monocytes

Dendritic cells
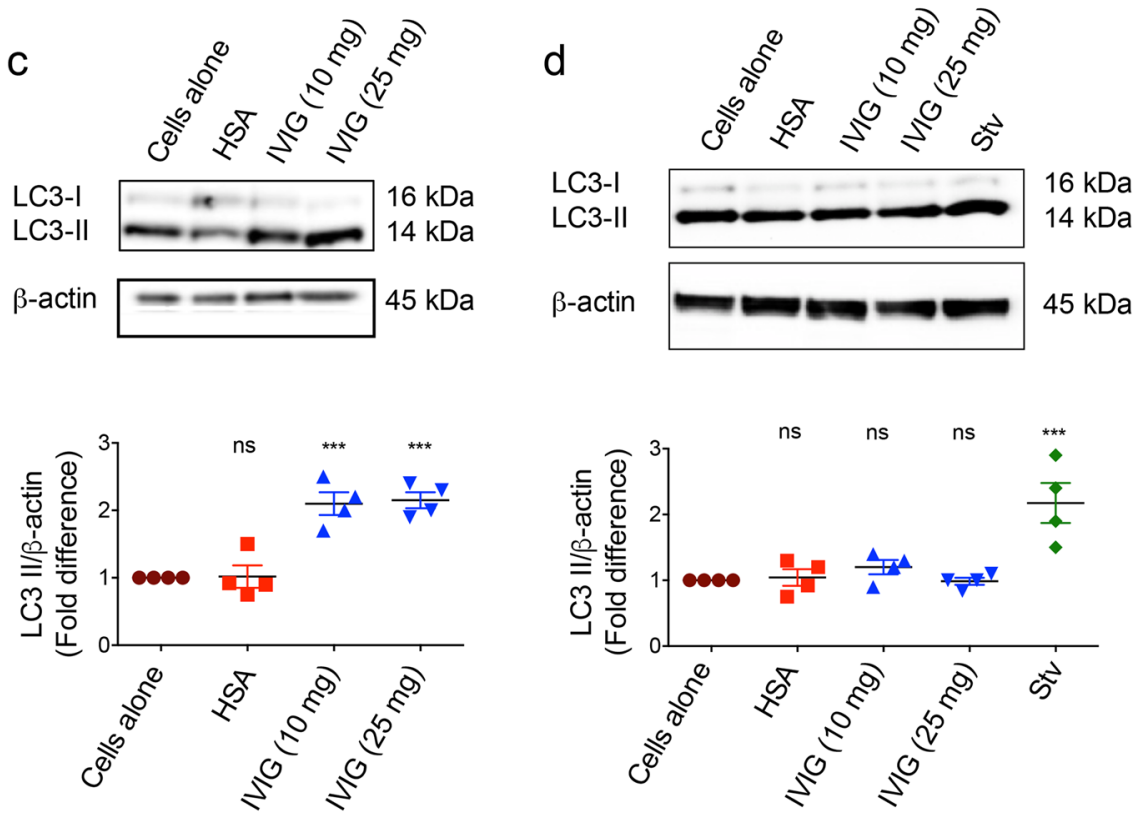

\section{M1 Macrophages}

M2 Macrophages

Fig. 2 IVIG induces autophagy in monocytes, dendritic cells and M1 macrophages but not in M2 macrophages. a-d Peripheral blood monocytes (a), monocyte-derived DCS (b), M1 macrophages (c) and M2 macrophages (d) were cultured with IVIG for 24 h. Levels of LC3-II and fold changes in the LC3-II levels (mean \pm SEM, $n=4$ donors) are presented. Starvation condition (Stv) was used as a positive control for autophagy. Images are cropped for the presentation. ${ }^{* *} P<0.01$; ${ }^{* *} P<0.001$; ns not significant; by one-way ANOVA with Dunnett's multiple comparison test.

Fig. 6c, cytochalasin D treatment abrogated IVIG-induced autophagy indicating that endocytosis of IVIG is mandatory for the induction of autophagy in innate cells like monocytes.

To confirm that loss of IVIG-induced autophagy upon cytochalasin D treatment was not due to its adverse effects on the autophagy machinery itself, we treated monocytes with rapamycin either alone or in combination with cytochalasin D. We found that rapamycin-induced autophagy was not affected by cytochalasin D (Fig. 6c), thus confirming that cytochalasin D specifically inhibited IVIG-induced autophagy. 


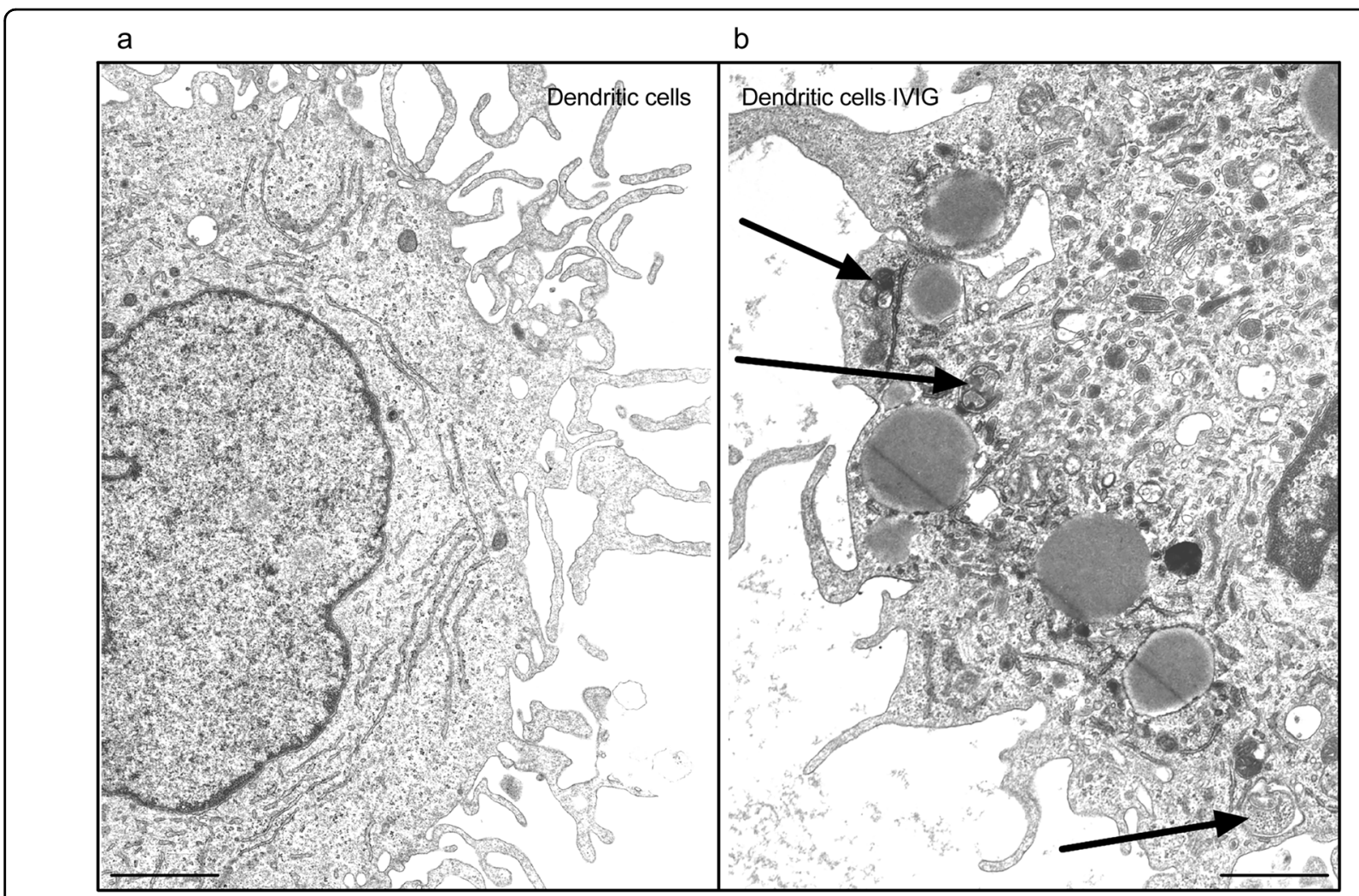

Fig. 3 Transmission electron microscopic analysis of autophagic organelles induced by IVIG in dendritic cells. a, b Lipopolysaccharidestimulated monocyte-derived human DCs were cultured for $24 \mathrm{~h}$ in the absence (a) or presence of IVIG (b). The cells were then processed for the transmission electron microscopy to visualize autophagic organelles. Arrows show autophagosomes. (scale bar $=100 \mathrm{~nm}$ ).

\section{Induction of autophagy is critical for the anti-inflammatory actions of IVIG}

After demonstrating that IVIG induces autophagy in various innate immune cells, we investigated if this process is critical for the anti-inflammatory actions of IVIG. By using lipopolysaccharide-stimulated human monocytederived DCs as a model, we found that the ability of IVIG to suppress the production of inflammatory cytokines like IL-8, IL-1 $\beta$ and IL- 6 were compromised when autophagy in DCs was inhibited through 3MA (Fig. $7 \mathrm{a}-\mathrm{c})^{36}$. These data thus show that induction of autophagy is integral to the anti-inflammatory mechanisms of IVIG.

\section{IVIG therapy induces autophagy in autoimmune patients}

Indispensability of autophagy in mediating antiinflammatory actions of IVIG raised an interesting perspective of whether this immunotherapy in autoimmune patients also induces autophagy. Earlier studies have indicated that IVIG therapy benefits patients with inflammatory myopathies ${ }^{17-19}$. Therefore, we investigated if IVIG therapy induces autophagy in these patients. PBMCs from the paired (pre- and post-IVIG therapy) blood samples of patients were subjected to western blot for the analysis of LC3-II. Prior to IVIG therapy, patients'
PBMCs showed minimal level of LC3-II. However, IVIG therapy led to significantly enhanced levels of LC3-II in the patients' PBMC (Fig. 8a, b), indicating that IVIG treatment induces autophagy in autoimmune patients thus providing translation insight on our results.

To corroborate these results, we analyzed several inflammatory cytokines like IL- 8 , TNF- $\alpha$, IL- $1 \beta$ and IL$17 \mathrm{~A}$ in the plasma of these inflammatory myopathy patients following IVIG therapy. In line with the wellknown anti-inflammatory effects of IVIG demonstrated in various pathologies, IVIG reduced the inflammatory cytokines in myopathy patients (Fig. 8c).

\section{Discussion}

Immunoglobulins are classically known for their protective role against infections. However, several lines of evidence also suggest that immunoglobulins are critical for mediating immune homeostasis by clearing apoptotic, altered and senescent cells, protecting the mucosal layers, and hence blunting the inflammation and autoimmune responses $^{37-39}$. Additional functions of circulating immunoglobulins and in particular IgG in immune homeostasis were comprehended through the therapeutic use of IVIG. As autophagy plays an important role in 

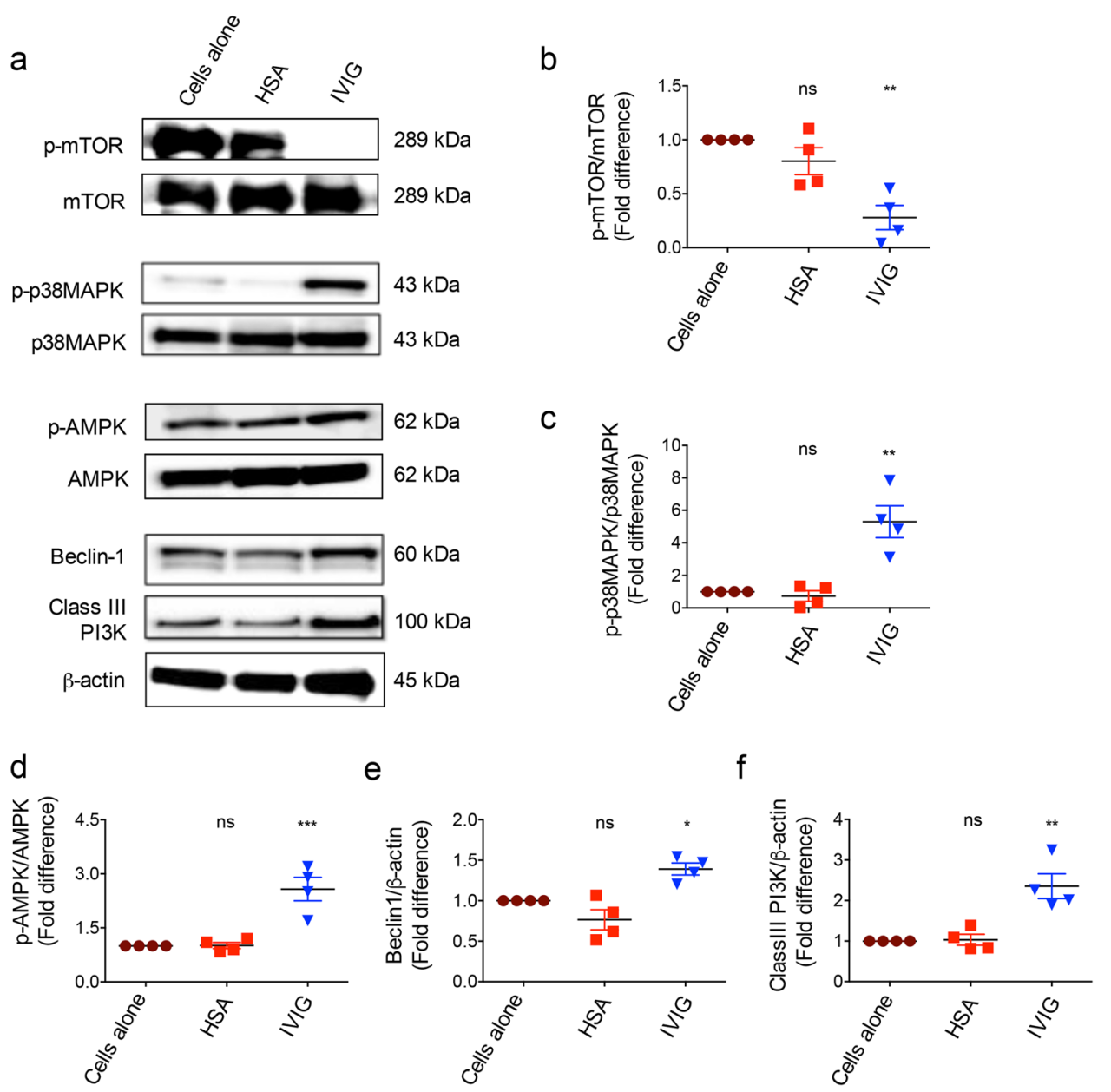

Fig. 4 IVIG-induced autophagy in monocytes is associated with induction of class III PI3K, beclin-1, p-AMPK and p-p38MAPK and inhibition of p-mTOR. a Monocytes were cultured with IVIG $(25 \mathrm{mg} / \mathrm{ml})$ or HSA for $24 \mathrm{~h}$. Expression of p-mTOR, mTOR, p-p38MAPK, p38MAPK, p-AMPK, AMPK, beclin-1 and class III PI3K was analyzed by western blot. Representative blots of four experiments by using monocytes from different donors are shown. $\mathbf{b}-\mathbf{f}$ Densitometry analysis of fold changes (mean $\pm \mathrm{SEM}, n=4)$ in the expression of p-mTOR (b), p-p38MAPK (c), p-AMPK (d), beclin-1 (e), and class III PI3K (f). ${ }^{*} P<0.05 ;{ }^{* *} P<0.01 ;{ }^{* * *} P<0.001$; ns not significant; as determined by one-way ANOVA Dunnett's multiple comparison test.

maintaining "healthy" status of the cell by clearing damaged organelles, cytotoxic molecules and misfolded proteins, we hypothesized that circulating normal IgG and hence IVIG exerts immune homeostasis via autophagy.

Several reports have demonstrated that cytokines play a key role in the regulation of autophagy in immune cells. Thus, proinflammatory cytokines including IFN- $\gamma$, TNF$\alpha$, IL-1 and IL-23 induce autophagy ${ }^{40}$, while Th2 and regulatory cytokines including IL-4, IL-13 and IL-10 are inhibitory ${ }^{41,42}$. Here, by using IVIG we provide compelling evidence that in addition to cytokines, the immunoglobulins that are normal constituents of a body potently regulate autophagy. As immunoglobulin deficiency is associated with high incidence of autoimmune and inflammatory diseases ${ }^{43}$, and that autophagy plays an important role in the regulation of autoimmune and autoinflammatory responses, our data suggest that immunoglobulins link autophagy to maintain immune homeostasis and to prevent immune aggression.

Autophagy is controlled by products of numerous Atg and several macromolecular signaling complexes including $\mathrm{mTOR}^{3}$. Inhibition of $\mathrm{mTOR}$ is essential for the initiation of autophagy and to allow the translocation of a complex containing Atg1/unc-51-like kinase1/ 2, Atg13, FIP200 and Atg101 from cytosol to the endoplasmic reticulum ${ }^{1}$. This leads to the recruitment of type III PI3K and VPS34 in a complex with other proteins, including beclin-1 to the developing autophagosome ${ }^{1,3}$. Rapamycin that inhibits mTOR and enhances autophagy has been successfully used in the treatment of rheumatoid arthritis ${ }^{44,45}$. In lupus-prone mice, rapamycin treatment prevents development of nephritis, inhibits lymphoproliferation, reduces autoantibody production and enhances the survival of 

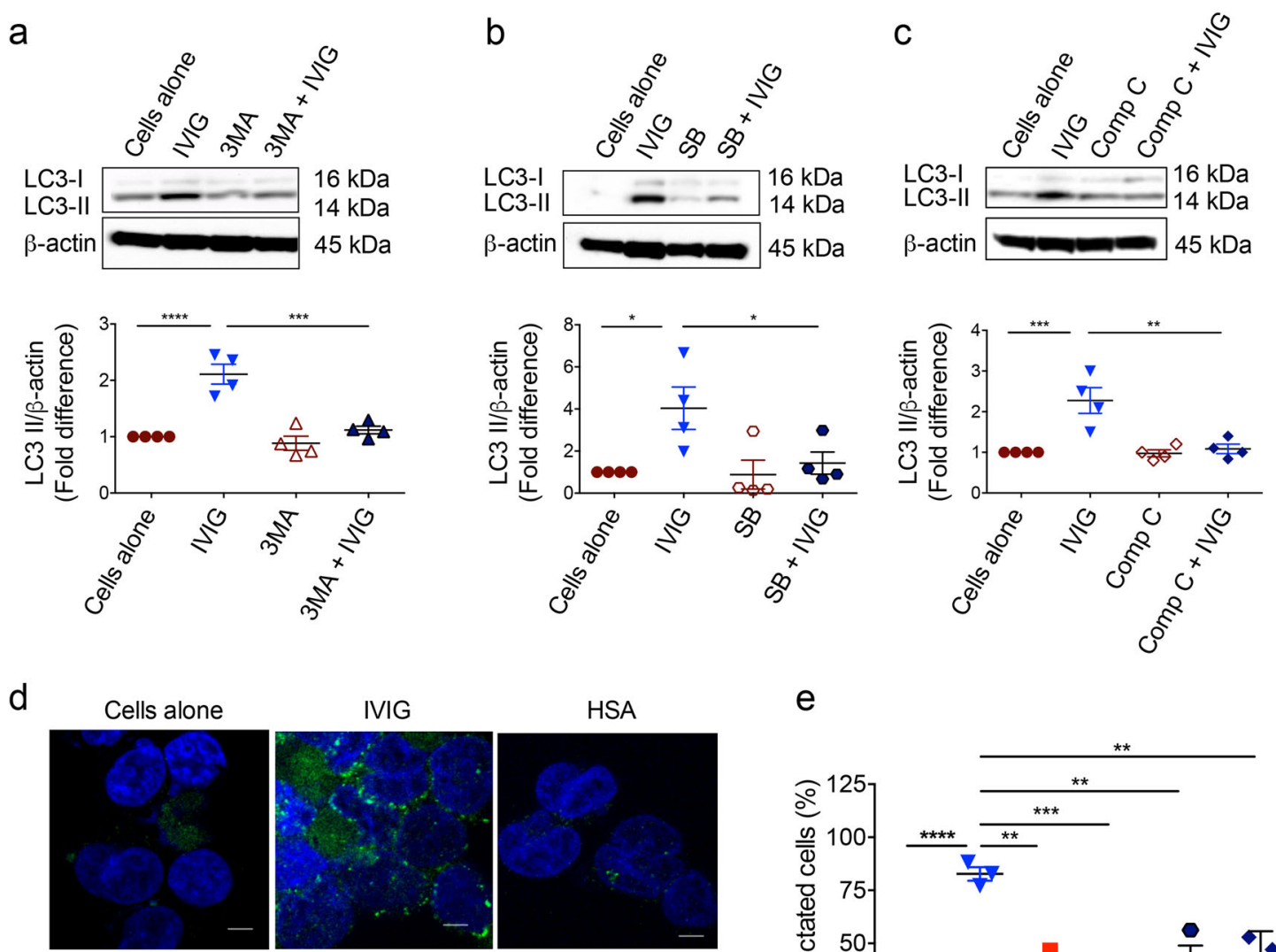

e
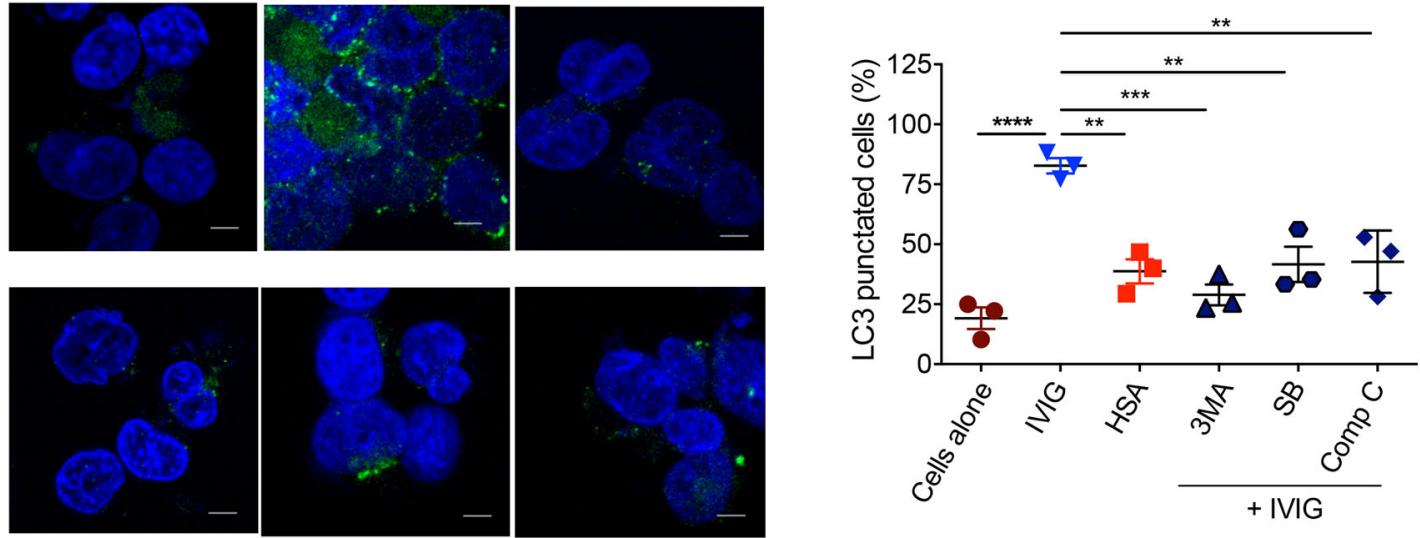

3MA

SB

Comp C

+ IVIG

Fig. 5 Inhibition of Class III PI3K, p38MAPK and AMPK kinases abrogates IVIG-induced autophagy in monocytes. a-c Peripheral blood monocytes were pretreated with a Class III PI3K inhibitor: 3MA, b p38MAPK inhibitor: SB202190 and c AMPK inhibitor: Compound C, followed by IVIG treatment for $24 \mathrm{~h}$. Levels of LC3-II by western blot and densitometry analyses of fold changes (mean \pm SEM; $n=4$ donors) are presented. Images are

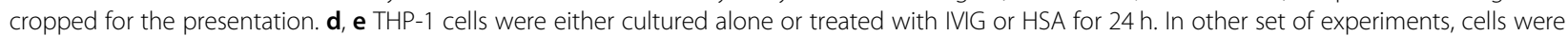
pretreated with respective inhibitors followed by IVIG treatment for $24 \mathrm{~h}$. The cells were then stained with anti-MAP1LC3I/II primary antibody followed by Alexa Fluor ${ }^{\circledast}$ 488-conjugated secondary antibody. Nuclei were stained with DAPI. Representative fluorescent images and percent (mean $\pm \mathrm{SEM} ; n=3)$ of LC3-punctated cells are shown. Bars indicate magnifications $(\times 63$. Scale bars $=5 \mu \mathrm{m}) .{ }^{*} P<0.05 ;{ }^{* *} P<0.01 ;{ }^{* * *} P<0.001 ;{ }^{* * * *} P<$ 0.0001; as determined by one-way ANOVA Dunnett's (a-c) or Tukey's multiple comparison test (e).

mice $^{46}$. Recent report in an experimental model also showed that preventive and curative treatment with rapamycin decreases the severity of myositis ${ }^{47}$. In view of these data from rapamycin and well-demonstrated beneficial effects of IVIG therapy in inflammatory myopathies led us to hypothesize that IVIG might induce autophagy in immune cells and hence represents a novel mechanism of action of IVIG in autoimmune and inflammatory conditions. In fact, our data show that IVIG therapy induces autophagy in immune cells and is associated with inhibition of mTOR phosphorylation.

IVIG has been demonstrated to modulate the functions of both innate and adaptive immune cells. Thus, IVIG suppresses the activation of various innate cells such as DCs, macrophages, and monocytes, and the secretion of inflammatory cytokines while enhancing anti- 

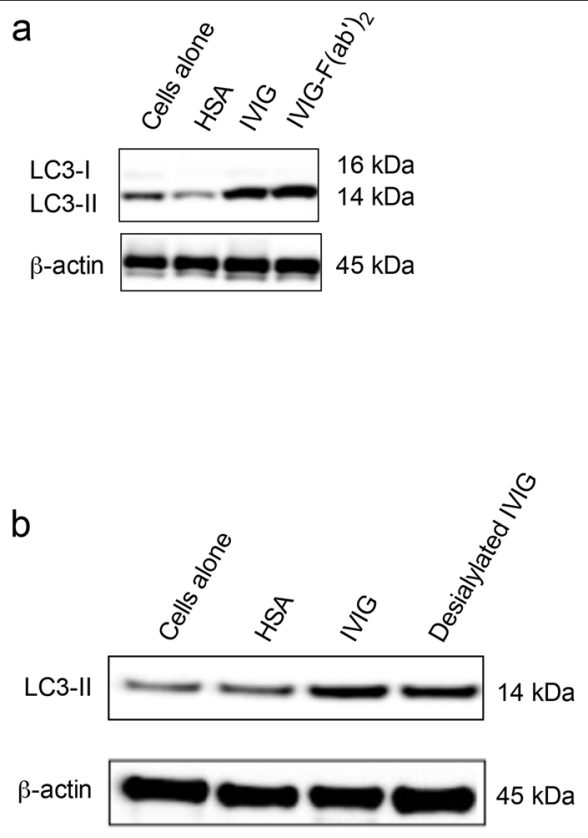

C

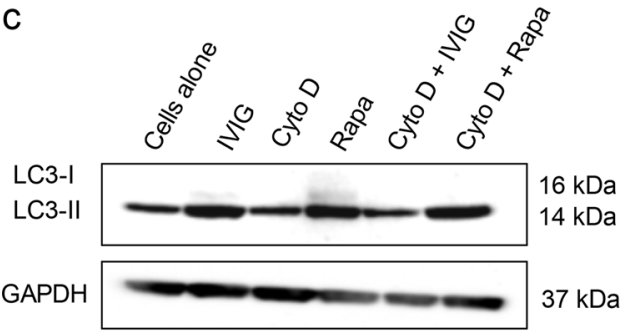

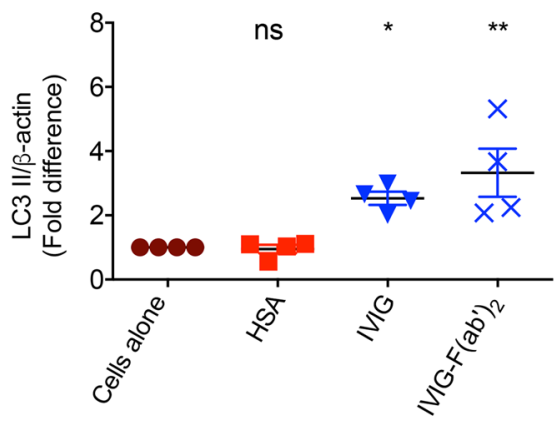
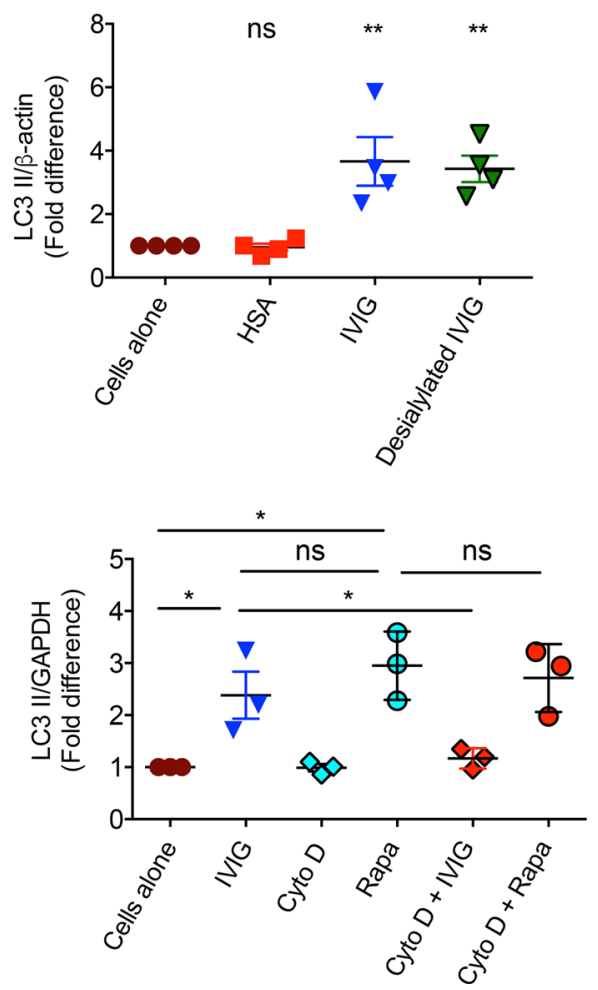

Fig. 6 IVIG-induced autophagy is $\mathrm{F}\left(\mathrm{ab}^{\prime}\right)_{2}$-dependent but sialylation-independent, and requires endocytosis of IgG. a, b Monocytes were cultured with IVIG $(25 \mathrm{mg} / \mathrm{ml})$, equimolar concentration of $F\left(\mathrm{ab}^{\prime}\right)_{2}$ or desialylated IVIG for $24 \mathrm{~h}$. Levels of LC3-II were analyzed by western blot. Representative blots of four different donors and fold changes in the LC3-II levels based on densitometry analysis of western blots (mean \pm SEM, $n=$ 4 donors) are presented. Images are cropped for the presentation. c Monocytes were pretreated with cytochalasin D (Cyto D) followed by IVIG or rapamycin (Rapa) treatment for $24 \mathrm{~h}$. Levels of LC3-II by western blot and densitometry analysis of fold changes (mean \pm SEM; $n=3$ donors) are shown. ${ }^{*} P<0.05$; ${ }^{*} P<0.01$; ns not significant; as determined by one-way ANOVA with Dunnett's multiple comparison test.

inflammatory mediators like IL-10 and IL-1RA ${ }^{21,48-52}$. The present data demonstrate that IVIG exerts autophagy in several inflammatory innate cells like monocytes, DCs and M1 macrophages but not in the cells associated with Th2 immune response like M2 macrophages. The inability of IVIG to induce autophagy in M2 macrophages might not be due to differences in the endocytosis activity of these innate cells. In fact, previous data have shown that M2 macrophages have more endocytic activity compared to M1 macrophages ${ }^{53}$. On the other hand, cytokines that are produced by M2 macrophages such as IL-13 and IL-10 are known to inhibit autophagy ${ }^{41,42}$. Also, autophagy machinery is intact in M2 macrophages as starvation condition was able to induce autophagy in M2 macrophages (Fig. 2d). All these evidences thus suggest that lack of induction of autophagy by IVIG in M2 macrophages is likely due to the cytokines produced by $\mathrm{M} 2$ macrophages.

In view of diverse role of autophagy in the regulation of inflammation and autoimmune responses, we propose 

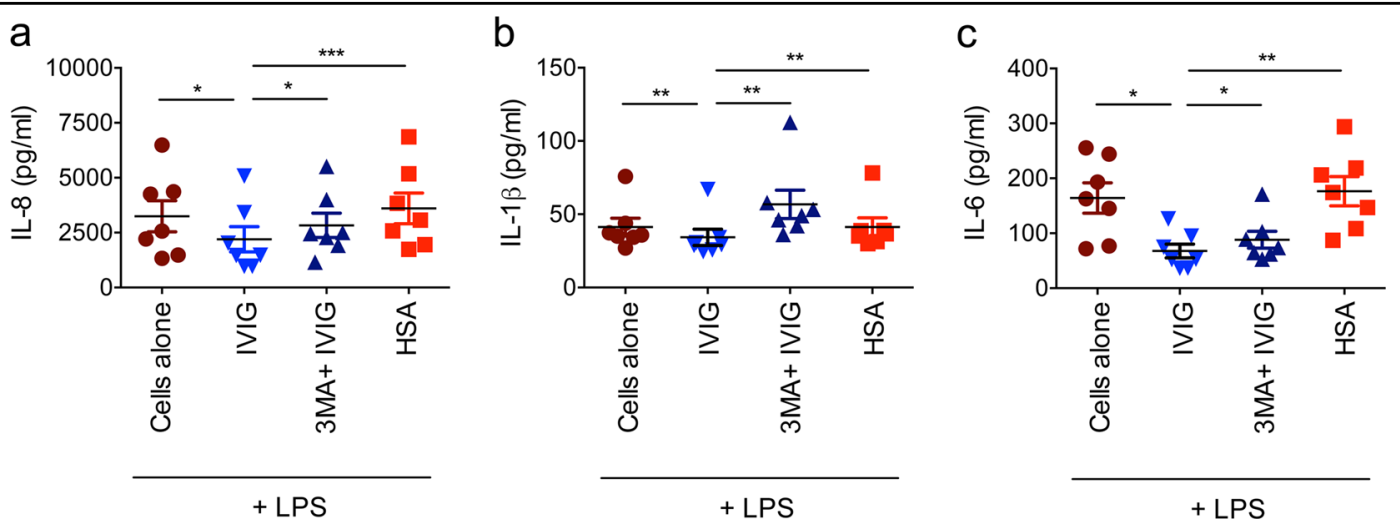

Fig. 7 Induction of autophagy is critical for the anti-inflammatory actions of IVIG. LPS-stimulated DCs were pretreated with 3MA followed by culture with IVIG $(25 \mathrm{mg} / \mathrm{ml})$ for $24 \mathrm{~h}$. Amounts of IL-8, IL-1 $\beta$ and IL-6 (mean \pm SEM; $n=7$ donors) in the cell-free supernatants were analyzed by ELISA. ${ }^{*} P<0.05 ;{ }^{* *} P<0.01$; ${ }^{* *} P<0.001$; as determined by one-way ANOVA with Tukey's multiple comparison test.

that autophagy is central to the anti-inflammatory actions of IVIG. In support of this argument, we found that the ability of IVIG to suppress the production of inflammatory cytokines is compromised when autophagy was inhibited. Although induction of autophagy by IVIG was higher at concentrations corresponding to high-dose therapy in autoimmune diseases, autophagy was significantly induced by IVIG even at concentrations corresponding to the circulating levels of IgG $(10 \mathrm{mg})$ in healthy individuals. As IVIG is a pool of IgG from the plasma of healthy individuals, these results thus point towards a role for circulating normal IgG in the maintenance of immune homeostasis.

While mTOR acts as negative regulator of autophagy, beclin-1 and several kinases like class III PI3K, p38 MAPK and AMPK are critical for initiating autophagy although several studies also suggest that p38 MAPK has dual role in autophagy ${ }^{54,55}$. In this regard, the role of AMPK is of particular importance as phosphorylation of AMPK under the conditions of low cellular energy and essential amino acid deprivation directly antagonizes mTOR leading to initiation of autophagy ${ }^{56,57}$. Emerging evidences imply that AMPK is a key modulator of immune response. AMPK could reduce the severity of inflammation and tissue damage in various models of autoimmune diseases $^{58,59}$. In this study, we show that IVIG-induced autophagy is associated with induction and activation of AMPK, beclin-1, class III PI3K and p38 MAPK. It is not clear at this stage whether inhibition of MTOR by IVIG is mediated via AMPK or due to direct suppressive effects on mTOR. However, abrogation of IVIG-induced autophagy upon inhibition of AMPK implies that mTOR inhibition by IVIG was not due to its direct effect on this molecule rather via AMPK-mediated process.

Exploration of mechanisms by which IVIG induces autophagy has revealed that $\mathrm{F}\left(\mathrm{ab}^{\prime}\right)_{2}$ fragments induce autophagy similar to intact IgG while desialylation of IgG had no repercussion. This indicates that glycosylation patterns of Fc-fragments including sialylation ${ }^{26,60}$ have no major role in IVIG-induced autophagy. Indeed, several F $\left(\mathrm{ab}^{\prime}\right)_{2}$ fragments-mediated functions of IVIG have been reported like neutralization of $\mathrm{C} 3 \mathrm{a}$ and $\mathrm{C} 5 \mathrm{a}$ anaphylatoxins and pathogenic autoantibodies, regulation of human DCs and granulocyte functions, inhibition of phagocytosis, expansion of human regulatory $\mathrm{T}$ cells and inhibition of Th17 responses ${ }^{61-68}$. However, we found that IVIG might not induce autophagy by signaling via cell surface receptors, as inhibition of endocytosis of IgG via cytochalasin D that inhibits phagocytosis and macropinocytosis abrogated IVIG-induced autophagy. Several reports have demonstrated that innate immune cells internalize $\mathrm{IVIG}^{28,34,35}$. As equivalent amount of $\mathrm{HSA}$ failed to induce autophagy, this rules out the possibility that IVIG-induced autophagy is due to the stress caused by accumulation of high amounts of proteins in the endosomes. As IVIG has been demonstrated to interact with various cytoplasmic antigens ${ }^{69}$, this suggests that IVIG might induce autophagy either by modifying the autophagy cargo or interacting with molecules that promote autophagy. Dependency of IVIG on $F\left(a b^{\prime}\right)_{2}$ fragments to induce autophagy also point towards this possibility.

Several lines of evidence show that IVIG is an effective therapy for inflammatory myopathies including dermatomyositis and polymyositis ${ }^{17-20,70}$. IVIG therapy response in inflammatory myopathy patients is associated with the modulation of various populations of immune cells and inflammatory mediators ${ }^{68,71-76}$. Our current data also demonstrate that IVIG therapy results in the induction of autophagy in PBMCs and downregulation of several inflammatory cytokines in the circulation including IL-8, TNF- $\alpha$, IL-1 $\beta$ and IL-17A. However, induction 


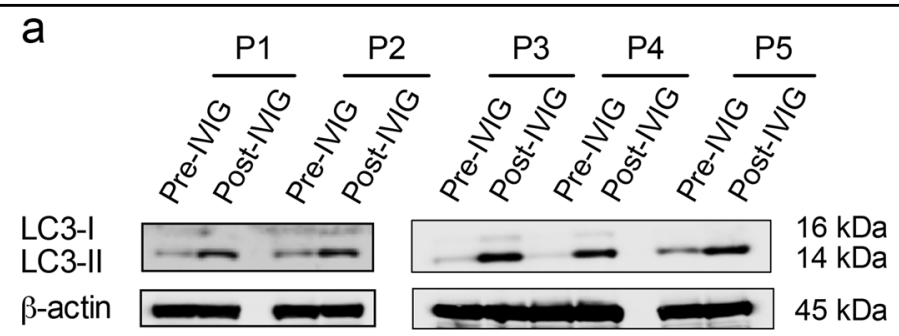

b

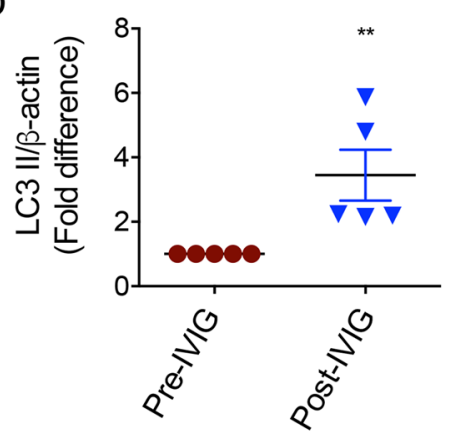

C
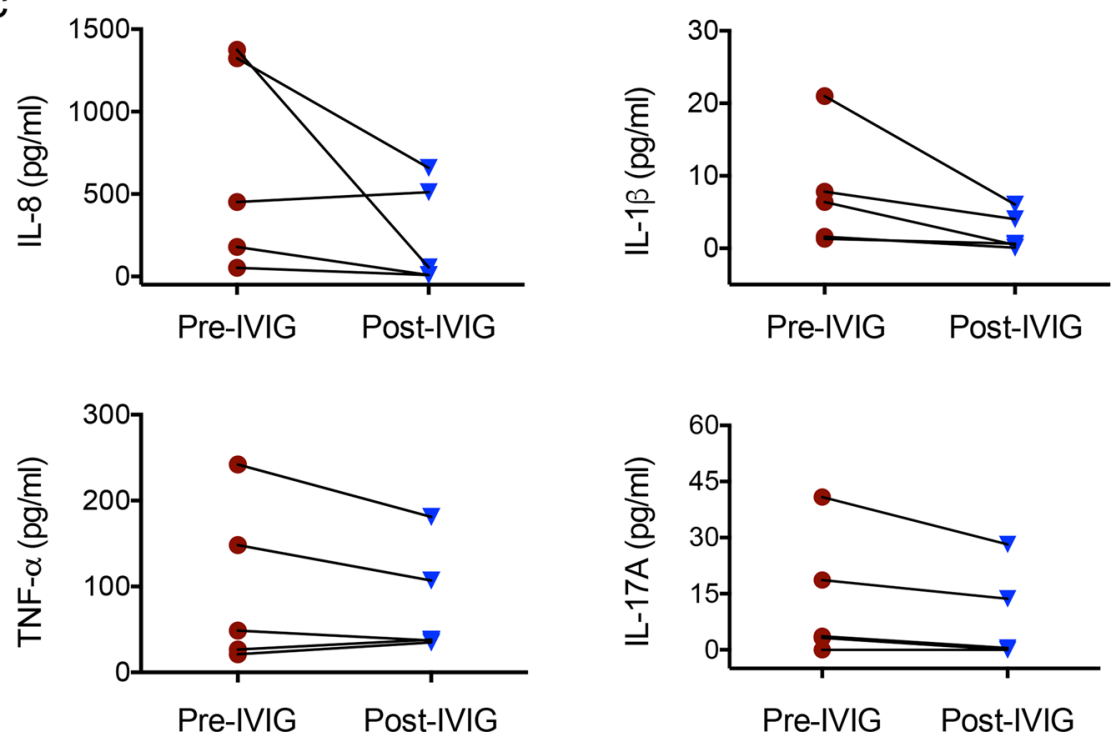

Fig. 8 IVIG therapy in inflammatory myopathy patients induces autophagy. a Levels of LC3-II in PBMC of myopathy patients before (pre-IVIG) and post-IVIG therapy as analyzed by western blot (five patients, P1-P5). b Fold changes in the LC3-II levels based on the densitometry analysis of western blots (mean $\pm \mathrm{SEM}, n=5$ patients) ${ }^{* *} P<0.01$ two-tailed Mann-Whitney test. c Changes in the levels (pg/ml) of inflammatory cytokines (IL-8, TNF- $a, I L-1 \beta$ and IL-17A) in the plasma of myopathy patients following IVIG therapy.

of autophagy might not be beneficial in all the pathologies. In inclusion body myositis (IBM), increased maturation of autophagosomes has been observed in muscle fibers but lysosomal proteolytic activity was decreased resulting in the enhanced accumulation of misfolded proteins ${ }^{13}$. Other muscular disorders like IBM associated with Paget's disease of the bone, fronto-temporal dementia and amyotrophic lateral sclerosis (IBMPFD/ALS), and Xlinked myopathy with excessive autophagy (XMEA) also show defective autophagosome maturation and enhanced autophagosome biogenesis ${ }^{77,78}$. Although it is not known whether IVIG could induce autophagy in the muscle fibers similar to immune cells demonstrated here, these reports provide a pointer on why IVIG has only limited therapeutic benefits in IBM patients.

To conclude, our study demonstrates that IVIG therapy induces autophagy in patients with inflammatory myopathies. Further investigations revealed that IVIG-induced autophagy is restricted to inflammatory cells like monocytes, DCs and M1 macrophages but not in cells associated with 
Th2 immune response like M2 macrophages. Mechanistically, IVIG induces autophagy by $\mathrm{F}\left(\mathrm{ab}^{\prime}\right)_{2}$-dependent process via activation of $\mathrm{p} 38$ MAPK, AMPK and class III PI3K and this requires endocytosis of IgG. Autophagy process constitutes an integral arm of anti-inflammatory mechanisms of IVIG. Our data thus provide insight on how circulating normal immunoglobulin maintains immune homeostasis and explains in part the mechanism by which IVIG therapy benefits patients with autoimmune and inflammatory diseases.

\section{Materials and methods \\ Reagents and antibodies}

IFN- $\gamma$, M-CSF and IL-13 were from R\&D Systems (Lille, France). CD14 and CD4 MicroBeads, GM-CSF and IL-4 were from Miltenyi Biotec (Paris, France). Rabbit MAbs to p-p38MAPK (Thr180/Tyr182, Clone 12FB), PI3K class III (Clone D9A5), Beclin-1 (Clone D40C5), $\beta$-actin (Clone 13E5, HRP-conjugated), GAPDH (Clone 14C10, HRPconjugated); affinity purified antibodies to p-mTOR (Ser2448, \#2971), mTOR (\#2972), LC3B (\#2775), pAMPK (Thr172, \#2531) AMPK (\#2532) p38MAPK (\#9212), HRP-linked anti-rabbit IgG (\#7074) were from Cell Signaling Technology (Ozyme, Saint Quentin Yvelines, France). Plasma-derived HSA was from Laboratoire Française de Biotechnologies (Les Ulis, France) and E. coli 055:B5 lipopolysaccharide was purchased from SigmaAldrich Chimie S.a.r.l (St. Quentin Fallavier, France).

\section{Therapeutic IVIG}

Sandoglobulin ${ }^{\circledast}$ (CSL Behring, Bern, Switzerland) was used throughout the study. IVIG was dialyzed for $18 \mathrm{~h}$ against large volumes of phosphate-buffered saline (PBS) followed by RPMI- 1640 at $4{ }^{\circ} \mathrm{C} \mathrm{f}$. F $\left(\mathrm{ab}^{\prime}\right)_{2}$ fragments were generated by digesting IVIG with pepsin (Sigma-Aldrich) at 50:1 ratio for $18 \mathrm{~h}$ in $0.2 \mathrm{M}$ sodium acetate buffer ( $\mathrm{pH} 4.1)$. F $\left(\mathrm{ab}^{\prime}\right)_{2}$ fragments were extensively dialyzed against sterile PBS followed by RPMI- 1640 medium at $4{ }^{\circ} \mathrm{C}$ and filtered through $0.22 \mu \mathrm{m}$ membrane. Purity of $\mathrm{F}\left(\mathrm{ab}^{\prime}\right)_{2}$ fragments were verified by SDS-PAGE and Coomassie blue staining.

For desialylation of IgG, IVIG (Hizentra ${ }^{\circledR}$, CSL Behring) was treated with recombinant neuraminidase (New England BioLabs, USA) as previously described ${ }^{29}$. By lectin blotting and reversed phase -HPLC, we confirmed the desialylation of IgG.

\section{Purification of peripheral blood mononuclear cells and monocytes}

Peripheral blood mononuclear cells were obtained from the buffy bags of healthy donors by Ficoll density gradient centrifugation. Buffy bags were purchased from Centre Necker-Cabanel, Etablissement Français du Sang, Paris, France. Ethical committee permission was obtained for the use of buffy bags of healthy donors (Institut National de la Santé et de la Recherche-EFS ethical committee convention 15/EFS/012). Monocytes were isolated from the PBMCs by positive selection using human CD14 MicroBeads. The cell purity was more than $97 \%$.

\section{Generation of DCs, M1 and M2 macrophages}

DCs were generated from monocytes by differentiating them in the presence of GM-CSF (1000 IU $/ 10^{6}$ cells) and IL-4 (500 IU $/ 10^{6}$ cells) for 6 days $^{79}$.

To obtain macrophages, monocytes were cultured in the presence of M-CSF ( $200 \mathrm{ng} / 10^{6}$ cells) for 6 days. These macrophages were further polarized into either M1 or M2 macrophages by culturing in the presence of lipopolysaccharide ( $200 \mathrm{ng} / 10^{6}$ cells) and IFN- $\gamma(40 \mathrm{ng} /$ $10^{6}$ cells), and IL-4 (40 ng/10 $0^{6}$ cells) and IL-13 (40 ng/10 cells) respectively for 3 additional days.

\section{Culturing of immune cells}

PBMCs, peripheral blood monocytes, monocyte-derived DCs or macrophages (M1 and M2) were cultured in RPMI- 1640 containing $10 \%$ fetal calf serum ( 0.5 million cells $/ \mathrm{ml}$ ) either alone or with various concentrations of IVIG preparations (10 and $25 \mathrm{mg} / \mathrm{ml}$; native or desialylated IVIG) or equimolar concentrations of $\mathrm{F}\left(\mathrm{ab}^{\prime}\right)_{2}$ fragments of IVIG for $24 \mathrm{~h}$ unless otherwise stated. All cells were treated with bafilomycin $(50 \mathrm{nM})$ for last $45 \mathrm{~min}$. The equimolar concentration of HSA $(0.15 \mathrm{mM})$ was used as an irrelevant protein control. Autophagy was analyzed by measuring the LC3-II levels by western blots or by immunofluorescence.

As a positive control for autophagy, immune cells were cultured in Hank's Balanced Salt Solution for $4 \mathrm{~h}$ to induce autophagy by starvation or were treated with $100 \mathrm{nM}$ rapamycin (Calbiochem, Merck Chimie SAS, Fontenay sous Bois, France) for $24 \mathrm{~h}$.

For visualizing the autophagic organelles in IVIGtreated DCs by transmission electron microscopy, monocyte-derived DCs were cultured for $24 \mathrm{~h}$ either with lipopolysaccharide alone or with IVIG $(25 \mathrm{mg} / \mathrm{ml})$ added $30 \mathrm{~min}$ post-lipopolysaccharide stimulation. DCs were washed with PBS and then fixed with $2.5 \%$ glutaraldehyde (in $0.1 \mathrm{M} \mathrm{PBS}, \mathrm{pH}$ 7.4) for $30 \mathrm{~min}$. Fixed DCs were processed for transmission electron microscopy by standard procedure $^{80}$ and cells were observed with a Zeiss EM 10 electron microscope (Carl Zeiss Microscopy GmbH, Jena, Germany)

For measuring the autophagy flux, $\mathrm{PBMCs}$ from healthy donors were cultured with IVIG $(25 \mathrm{mg} / \mathrm{ml})$ for 0,18 , or $24 \mathrm{~h}$. At the end of treatment, cells were treated with or without bafilomycin (Baf) for $45 \mathrm{~min}$. Autophagy flux ( $\triangle \mathrm{LC} 3-\mathrm{II})$ was quantified by subtracting the band intensity of LC3-II in the presence of Baf from that of absence 
of Baf by using the formula:

$$
\Delta \mathrm{LC} 3-\mathrm{II}=[\mathrm{LC} 3-\mathrm{II}(+\mathrm{Baf})]-[\mathrm{LC} 3-\mathrm{II}(-\mathrm{Baf})]
$$

\section{Treatment of monocytes with pharmaceutical inhibitors}

Different pharmaceutical inhibitors, i.e., 3-methyladenine (3MA, for PI3K class III), SB202190 (for p38MAPK) and compound $\mathrm{C}$ (Comp C, for AMPK) were used in this study. Cytochalasin D was used to block endocytosis. The concentration of inhibitors was chosen after careful titration experiments assessing the viability of the monocytes. Monocytes were pretreated with 3MA ( 1 and $5 \mathrm{mM}$, for western blot and cytokine analyses respectively), SB202190 $(10 \mu \mathrm{M})$, compound $\mathrm{C}(5 \mu \mathrm{M})$ and cytochalasin $\mathrm{D}(10 \mu \mathrm{M})$ for $1 \mathrm{~h}$ with inhibitors followed by culture with IVIG for $24 \mathrm{~h}$. The cells were then processed for immunoblot.

\section{Inflammatory myopathy patients}

Heparinized blood samples of patients with inflammatory myopathies were obtained before and $24-48 \mathrm{~h}$ following initiation of IVIG $(2 \mathrm{~g} / \mathrm{kg})$ therapy. Informed consent was obtained from the patients. PBMCs were isolated by Ficoll density gradient centrifugation and processed for immunoblot. We analyzed five myopathy patients (two immune-mediated necrotizing myopathy patients, two dermatomyositis patients and one antisynthetase syndrome patient) with a median age of 48 years (ranging from 38 to 64) and include two men (Supplementary Table S1). The study was approved by CPP-Ile-de-France VI, Groupe Hospitalier Pitié-Salpêtrière, Paris. IgG levels in the plasma were measured by immunonephelometry (Cobas 6000 Roche Diagnostics).

\section{Measurement of inflammatory cytokines}

Lipopolysaccharide (100 ng/0.5 million cells $/ \mathrm{ml}$ )-stimulated DCs were treated with 3MA $(5 \mathrm{mM})$ for $1 \mathrm{~h}$ followed by culture with IVIG $(25 \mathrm{mg} / \mathrm{ml})$ for $24 \mathrm{~h}$. Amounts of DC-cytokines (IL-8, IL-6 and IL-1 $\beta$ ) in the cell-free supernatants and cytokines in the plasma of inflammatory myopathy patients (IL- 8 , TNF- $\alpha$, IL- $1 \beta$ and IL-17A) were analyzed by ELISA (ELISA Ready-SET-Go, eBioscience, Paris, France).

\section{Immunoblotting analysis}

Treated cells were pelleted and lysed in RIPA buffer (Sigma) containing protease and phosphatase inhibitors (Roche Biotech) on ice for $30 \mathrm{~min}$. Whole-cell lysates were collected. Proteins were quantified and equal amount of proteins from each sample was separated on SDS-PAGE. The proteins were then transferred onto polyvinylidene fluoride membranes by a wet western blotting method. The membrane was blocked using 5\% BSA in TBST $(20 \mathrm{mM}$ Tris- $\mathrm{HCl}$ [pH 7.4], $137 \mathrm{mM} \mathrm{NaCl}$, and $0.1 \%$
Tween 20) for $60 \mathrm{~min}$. The blots were incubated overnight at $4{ }^{\circ} \mathrm{C}$ with primary antibody diluted in TBST $-5 \%$ bovine serum albumin (BSA) followed by incubation with HRPconjugated anti-rabbit IgG secondary antibody for $2 \mathrm{~h}$. After washing in TBST, blots were developed with SuperSignal $^{\mathrm{TM}}$ WestDura Extended Duration substrate (ThermoFisher Scientific, Courtaboeuf, France) according to the manufacturer's instructions. $\beta$-actin or GAPDH was used as loading control. The western blot images were analyzed by myImageAnalysis software $\mathrm{v} \quad 2.0$ (ThermoFisher Scientific). Full western blot images are presented in Supplementary Fig. S1.

\section{Cell line}

THP-1, a pro-monocytic cell line (obtained from the National Center for Cell Sciences, Pune, India) was maintained in RPMI-1640 medium containing 10\% fetal bovine serum (FBS). Cells $(0.5$ million cells $/ \mathrm{ml})$ were treated with IVIG $(25 \mathrm{mg} / \mathrm{ml})$ or equimolar concentrations of HSA for $24 \mathrm{~h}$. In case of inhibitor treatment, cells were pretreated with inhibitors for $1 \mathrm{~h}$ followed by culture with IVIG for $24 \mathrm{~h}$. Cells were treated with bafilomycin for last $45 \mathrm{~min}$.

\section{Immunofluorescence}

Treated THP-1 cells were collected, fixed with $4 \%$ paraformaldehyde and washed with PBS. After blocking with blocking buffer $(1 \times$ PBS, $5 \%$ FBS and $0.3 \%$ Triton X100), cells were stained at $4{ }^{\circ} \mathrm{C}$ with polyclonal affinity purified rabbit anti-MAP1LC3I/II antibody (\#4108, Cell Signaling Technology) diluted in antibody dilution buffer (1× PBS, $1 \%$ BSA and $0.3 \%$ Triton X-100) for overnight. Cells were washed thoroughly three times and incubated with goat anti-rabbit IgG $(\mathrm{H}+\mathrm{L}), \mathrm{F}\left(\mathrm{ab}^{\prime}\right)_{2}$ fragment (Alexa Fluor $^{\circledR} 488$ conjugate, Cell Signaling Technology, \#4412) for $2 \mathrm{~h}$. The nuclei were stained with 4',6-Diamidino-2phenylindole dihydrochloride (DAPI, Sigma-Aldrich) for $10 \mathrm{~min}$. Cells were spread on coverslip and mounted with glycerol as a medium. Confocal images were taken using Zeiss LSM 710 meta confocal laser scanning microscope using a Plan-Achromat $\times 63 / 1.4$ oil DIC objective (both from Carl Zeiss). Images were analyzed using ZEN 2009 software.

\section{Statistical analysis}

As detailed in the figure legends, each experiment was repeated independently 3-7 times using different donors, except for autophagy flux that was repeated twice. Sample size was chosen based on the previous experience with western blot and biological assays, and literature survey. We did not use any specific test to ensure adequate power. The data were presented as mean \pm standard error of the mean (SEM) unless otherwise specified. Randomization and blinding were not used in the study. Statistical analyses were 
performed by two-way nonparametric Mann-Whitney test or one-way ANOVA (Dunnett's or Tukey's multiple comparison tests) as indicated in the figure legends using Prism 6 software. Values were deliberated statistically significant with ${ }^{*} P<0.05$, ${ }^{* *} P<0.01$, ${ }^{* * * *} P<0.001$, and ${ }^{* * * * *} P<0.0001$.

\section{Acknowledgements}

We thank Dr. L. Galluzzi, Dr. F. van de Veerdonk, Dr. F. Pietrocola and Dr. S. Holla for the suggestions and Dr. A. Bousquet for the analyses of IgG in the plasma. This work was supported by Institut National de la Santé et de la Recherche Médicale (INSERM), Sorbonne Université, Université Paris Descartes and CSL Behring, France; Department of Biotechnology, Department of Science and Technology (DST), DST-FIST, India. M.D. was recipient of Raman-Charpak fellowship from Indo-French Center for Promotion of Advanced Research (CEFIPRA). E.S-V. and A.K were recipient of doctoral fellowships from CEFIPRA. A.K. also received fellowship from La Fondation pour la Recherche Médicale (FDT201805005552). B.B. received fellowship from University Grant Commission, India. K.N.B. is a J.C. Bose National Fellow of DST, India.

\section{Author details}

${ }^{1}$ Institut National de la Santé et de la Recherche Médicale; Centre de Recherche des Cordeliers, Equipe- Immunopathologie et Immunointervention Thérapeutique, Sorbonne Université, 75006 Paris, France. ${ }^{2}$ Département de Médecine Interne et Immunologie Clinique, Hôpital Pitié-Salpêtrière, AP-HP, 75013 Paris, France. ${ }^{3}$ Department of Microbiology and Cell Biology, Indian Institute of Science, Bangalore 560012, India. ${ }^{4}$ Université Paris Descartes, Sorbonne Paris Cité, 75006 Paris, France. ${ }^{5}$ CSL Behring, Research, CSL Biologics Research Center, 3014 Bern, Switzerland. 'Service d'anatomie pathologique, Hôpital Européen Georges Pompidou, 75015 Paris, France. IInstitut National de la Santé et de la Recherche Médicale Unité 974, Sorbonne Université, 75013 Paris, France

\section{Conflict of interest}

The authors declare that they have no conflict of interest.

\section{Publisher's note}

Springer Nature remains neutral with regard to jurisdictional claims in published maps and institutional affiliations.

Supplementary Information accompanies this paper at (https://doi.org/ 10.1038/s41419-020-2249-y).

Received: 26 September 2019 Revised: 8 January 2020 Accepted: 9 January 2020

Published online: 23 January 2020

\section{References}

1. He, C. \& Klionsky, D. J. Regulation mechanisms and signaling pathways of autophagy. Annu. Rev. Genet. 43, 67-93 (2009).

2. Klionsky, D. J. et al. Guidelines for the use and interpretation of assays for monitoring autophagy (3rd edition). Autophagy 12, 1-222 (2016).

3. Mehrpour, M., Esclatine, A., Beau, I. \& Codogno, P. Overview of macroautophagy regulation in mammalian cells. Cell Res. 20, 748-762 (2010).

4. Bhattacharya, A. \& Eissa, N. T. Autophagy and autoimmunity crosstalks. Front. Immunol. 4, 88 (2013).

5. Pua, H. H., Dzhagalov, I., Chuck, M., Mizushima, N. \& He, Y. W. A critical role for the autophagy gene Atg5 in T cell survival and proliferation. J. Exp. Med. 204 25-31 (2007).

6. Rockel, J. S. \& Kapoor, M. Autophagy: controlling cell fate in rheumatic diseases. Nat. Rev. Rheumatol. 12, 517-531 (2016).

7. Gros, F. et al. Macroautophagy is deregulated in murine and human lupus T lymphocytes. Autophagy 8, 1113-1123 (2012).

8. Hampe, J. et al. A genome-wide association scan of nonsynonymous SNPS identifies a susceptibility variant for Crohn disease in ATG16L1. Nat. Genet. 39 207-211 (2007).
9. Rioux, J. D. et al. Genome-wide association study identifies new susceptibility loci for Crohn disease and implicates autophagy in disease pathogenesis. Nat. Genet. 39, 596-604 (2007).

10. Ramos, P. S. et al. A comprehensive analysis of shared loci between systemic lupus erythematosus (SLE) and sixteen autoimmune diseases reveals limited genetic overlap. PLoS Genet. 7, e1002406 (2011).

11. Lin, N. Y. et al. Autophagy regulates TNFa-mediated joint destruction in experimental arthritis. Ann. Rheum. Dis. 72, 761-768 (2013).

12. Alirezaei, M. et al. Elevated ATG5 expression in autoimmune demyelination and multiple sclerosis. Autophagy 5, 152-158 (2009).

13. Nogalska, A., D'Agostino, C., Terracciano, C., Engel, W. K. \& Askanas, V. Impaired autophagy in sporadic inclusion-body myositis and in endoplasmic reticulum stress-provoked cultured human muscle fibers. Am. J. Pathol. 177, 1377-1387 (2010).

14. Villella, V. R. et al. Autophagy suppresses the pathogenic immune response to dietary antigens in cystic fibrosis. Cell Death Dis. 10, 258 (2019).

15. Yin, $\mathrm{H}$. et al. The therapeutic and pathogenic role of autophagy in autoimmune diseases. Front. Immunol. 9, 1512 (2018).

16. Bonam, S. R., Wang, F. \& Muller, S. Autophagy: a new concept in autoimmunity regulation and a novel therapeutic option. J. Autoimmun. 94, 16-32 (2018).

17. Gelfand, E. W. Intravenous immune globulin in autoimmune and inflammatory diseases. N. Engl. J. Med. 367, 2015-2025 (2012).

18. Bayry, J., Negi, V. S. \& Kaveri, S. V. Intravenous immunoglobulin therapy in rheumatic diseases. Nat. Rev. Rheumatol. 7, 349-359 (2011).

19. Perez, E. E. et al. Update on the use of immunoglobulin in human disease: a review of evidence. J. Allergy Clin. Immunol. 139, S1-S46 (2017).

20. Lunemann, J. D., Nimmerjahn, F. \& Dalakas, M. C. Intravenous immunoglobulin in neurology — mode of action and clinical efficacy. Nat. Rev. Neurol. 11, 80-89 (2015).

21. Galeotti, C., Kaveri, S. V. \& Bayry, J. IVIG-mediated effector functions in autoimmune and inflammatory diseases. Int. Immunol. 29, 491-498 (2017).

22. Seite, J. F., Shoenfeld, Y., Youinou, P. \& Hillion, S. What is the contents of the magic draft IVlg? Autoimmun. Rev. 7, 435-439 (2008).

23. Seite, J. F. Hillion, S., Harbonnier, T. \& Pers, J. O. Review: intravenous immunoglobulin and $B$ cells: when the product regulates the producer. Arthritis Rheumatol. 67, 595-603 (2015).

24. Maddur, M. S., Kaveri, S. V. \& Bayry, J. Circulating normal lgG as stimulator of regulatory $T$ cells: lessons from intravenous immunoglobulin. Trends Immunol. 38, 789-792 (2017)

25. Kabeya, Y. et al. LC3, a mammalian homologue of yeast Apg8p, is localized in autophagosome membranes after processing. EMBO J. 19, 5720-5728 (2000).

26. Fiebiger, B. M., Maamary, J., Pincetic, A. \& Ravetch, J. V. Protection in antibodyand $T$ cell-mediated autoimmune diseases by antiinflammatory IgG FCS requires type II FcRs. Proc. Natl Acad. Sci. USA 112, E2385-E2394 (2015).

27. Schwab, I. et al. Broad requirement for terminal sialic acid residues and FcyRlIB for the preventive and therapeutic activity of intravenous immunoglobulins in vivo. Eur. J. Immunol. 44, 1444-1453 (2014).

28. Massoud, A. H. et al. Dendritic cell immunoreceptor: a novel receptor for intravenous immunoglobulin mediates induction of regulatory $T$ cells. J. Allergy Clin. Immunol. 133, 853-863 (2014). e855.

29. Bozza, S., Kasermann, F., Kaveri, S. V., Romani, L. \& Bayry, J. Intravenous immunoglobulin protects from experimental allergic bronchopulmonary aspergillosis via a sialylation-dependent mechanism. Eur. J. Immunol. 49, 195-198 (2019).

30. Othy, S. et al. Sialylation may be dispensable for reciprocal modulation of helper T cells by intravenous immunoglobulin. Eur. J. Immunol. 44, 2059-2063 (2014).

31. Nagelkerke, S. Q. et al. Inhibition of FcyR-mediated phagocytosis by IVlg is independent of IgG-Fc sialylation and FcyRllb in human macrophages. Blood 124, 3709-3718 (2014).

32. Campbell, I. K. et al. Therapeutic effect of IVIG on inflammatory arthritis in mice is dependent on the Fc portion and independent of sialylation or basophils. J. Immunol. 192, 5031-5038 (2014).

33. Leontyev, D. et al. Sialylation-independent mechanism involved in the amelioration of murine immune thrombocytopenia using intravenous gammaglobulin. Transfusion 52, 1799-1805 (2012).

34. Tha-In, T. et al. Intravenous immunoglobulins suppress T-cell priming by modulating the bidirectional interaction between dendritic cells and natura killer cells. Blood 110, 3253-3262 (2007). 
35. Trepanier, P., Aubin, E. \& Bazin, R. IVlg-mediated inhibition of antigen presentation: predominant role of naturally occurring cationic lgG. Clin. Immunol. 142, 383-389 (2012)

36. Blommaart, E. F., Krause, U., Schellens, J. P., Vreeling-Sindelarova, H. \& Meijer, A. J. The phosphatidylinositol 3-kinase inhibitors wortmannin and LY294002 inhibit autophagy in isolated rat hepatocytes. Eur. J. Biochem. 243, 240-246 (1997).

37. Ehrenstein, M. R. \& Notley, C. A. The importance of natural lgM: scavenger, protector and regulator. Nat. Rev. Immunol. 10, 778-786 (2010).

38. Kaveri, S. V., Silverman, G. J. \& Bayry, J. Natural lgM in immune equilibrium and harnessing their therapeutic potential. J. Immunol. 188, 939-945 (2012).

39. Panda, S. \& Ding, J. L. Natural antibodies bridge innate and adaptive immunity. J. Immunol. 194, 13-20 (2015).

40. Peral de Castro, C. et al. Autophagy regulates IL-23 secretion and innate T cell responses through effects on IL-1 secretion. J. Immunol. 189, 4144-4153 (2012).

41. Harris, J. et al. T helper 2 cytokines inhibit autophagic control of intracellular Mycobacterium tuberculosis. Immunity 27, 505-517 (2007).

42. Park, H. J. et al. IL-10 inhibits the starvation induced autophagy in macrophages via class I phosphatidylinositol 3-kinase (PI3K) pathway. Mol. Immunol. 48, 720-727 (2011).

43. Fischer, A. et al. Autoimmune and inflammatory manifestations occur frequently in patients with primary immunodeficiencies. J. Allergy Clin. Immunol. 140, 1388-1393 (2017). e1388.

44. Laragione, T. \& Gulko, P. S. mTOR regulates the invasive properties of synovial fibroblasts in rheumatoid arthritis. Mol. Med. 16, 352-358 (2010).

45. Cejka, D. et al. Mammalian target of rapamycin signaling is crucial for joint destruction in experimental arthritis and is activated in osteoclasts from patients with rheumatoid arthritis. Arthritis Rheum. 62, 2294-2302 (2010).

46. Ramos-Barron, A. et al. Prevention of murine lupus disease in (NZBXNZW)F1 mice by sirolimus treatment. Lupus 16, 775-781 (2007).

47. Prevel, N., Allenbach, Y., Klatzmann, D., Salomon, B. \& Benveniste, O. Beneficial role of rapamycin in experimental autoimmune myositis. PLOS ONE 8, e74450 (2013).

48. Bayry, J. et al. Intravenous immunoglobulin abrogates dendritic cell differentiation induced by interferon-a present in serum from patients with systemic lupus erythematosus. Arthritis Rheum. 48, 3497-3502 (2003).

49. Siragam, V. et al. Intravenous immunoglobulin ameliorates ITP via activating FC gamma receptors on dendritic cells. Nat. Med. 12, 688-692 (2006).

50. Smed-Sorensen, A. et al. IgG regulates the CD1 expression profile and lipid antigen-presenting function in human dendritic cells via FcyRlla. Blood $\mathbf{1 1 1}$ 5037-5046 (2008).

51. Kozicky, L. K. et al. Intravenous immunoglobulin skews macrophages to an anti-inflammatory, IL-10-producing activation state. J. Leukoc. Biol. 98, 983-994 (2015).

52. Fann, D. Y. et al. Intravenous immunoglobulin suppresses NLRP1 and NLRP3 inflammasome-mediated neuronal death in ischemic stroke. Cell Death Dis. 4, e790 (2013)

53. Tarique, A. A. et al. Phenotypic, functional, and plasticity features of classical and alternatively activated human macrophages. Am. J. Respir. Cell Mol. Biol. 53, 676-688 (2015).

54. Webber, J. L. Regulation of autophagy by p38alpha MAPK. Autophagy 6 , 292-293 (2010)

55. Kim, D. S. et al. p38 Mitogen-activated protein kinase is involved in endoplasmic reticulum stress-induced cell death and autophagy in human gingival fibroblasts. Biol. Pharm. Bull. 33, 545-549 (2010).

56. Kim, J., Kundu, M., Viollet, B. \& Guan, K. L. AMPK and mTOR regulate autophagy through direct phosphorylation of Ulk1. Nat. Cell. Biol. 13, 132-141 (2011).

57. Shang, L. et al. Nutrient starvation elicits an acute autophagic response mediated by Ulk1 dephosphorylation and its subsequent dissociation from AMPK. Proc. Natl Acad. Sci. USA 108, 4788-4793 (2011).

58. Bai, A. et al. AMPK agonist downregulates innate and adaptive immune responses in TNBS-induced murine acute and relapsing colitis. Biochem. Pharmacol. 80, 1708-1717 (2010).

59. Nath, N. et al. Loss of AMPK exacerbates experimental autoimmune encephalomyelitis disease severity. Biochem. Biophys. Res. Commun. 386, 16-20 (2009).
60. Anthony, R. M., Kobayashi, T., Wermeling, F. \& Ravetch, J. V. Intravenous gammaglobulin suppresses inflammation through a novel $T(H) 2$ pathway. Nature 475, 110-113 (2011).

61. Shoenfeld, Y. et al. Efficacy of IVIG affinity-purified anti-double-stranded DNA anti-idiotypic antibodies in the treatment of an experimental murine model of systemic lupus erythematosus. Int. Immunol. 14, 1303-1311 (2002).

62. Maddur, M. S. et al. Inhibition of differentiation, amplification, and function of human TH17 cells by intravenous immunoglobulin. J. Allergy Clin. Immunol. 127, 823-830 (2011). e821-827.

63. Trinath, J. et al. Intravenous immunoglobulin expands regulatory $T$ cells via induction of cyclooxygenase-2-dependent prostaglandin E2 in human dendritic cells. Blood 122, 1419-1427 (2013).

64. Othy, S. et al. Intravenous gammaglobulin inhibits encephalitogenic potential of pathogenic T cells and interferes with their trafficking to the central nervous system, implicating sphingosine-1 phosphate receptor 1-mammalian target of rapamycin axis. J. Immunol. 190, 4535-4541 (2013).

65. Wiedeman, A. E. et al. Contrasting mechanisms of interferon-a inhibition by intravenous immunoglobulin after induction by immune complexes versus Toll-like receptor agonists. Arthritis Rheum. 65, 2713-2723 (2013)

66. Saha, C. et al. Monomeric immunoglobulin A from plasma inhibits human Th17 responses in vitro independent of FcaRI and DC-SIGN. Front. Immunol. 8, 275 (2017).

67. Schneider, C. et al. IVIG regulates the survival of human but not mouse neutrophils. Sci. Rep. 7, 1296 (2017).

68. Galeotti, C. et al. Intravenous immunoglobulin induces IL-4 in human basophils by signaling through surface-bound IgE. J. Allergy Clin. Immunol. 144, 524-535 (2019). e528.

69. Rossi, F., Jayne, D. R., Lockwood, C. M. \& Kazatchkine, M. D. Anti-idiotypes against anti-neutrophil cytoplasmic antigen autoantibodies in normal human polyspecific lgG for therapeutic use and in the remission sera of patients with systemic vasculitis. Clin. Exp. Immunol. 83, 298-303 (1991).

70. Dalakas, M. C. et al. A controlled trial of high-dose intravenous immune globulin infusions as treatment for dermatomyositis. N. Engl. J. Med. 329, 1993-2000 (1993).

71. Basta, M. \& Dalakas, M. C. High-dose intravenous immunoglobulin exerts its beneficial effect in patients with dermatomyositis by blocking endomysial deposition of activated complement fragments. J. Clin. Invest. 94, 1729-1735 (1994).

72. Tournadre, A. et al. Th1 and Th17 balance in inflammatory myopathies: interaction with dendritic cells and possible link with response to high-dose immunoglobulins. Cytokine 46, 297-301 (2009).

73. Bayry, J., Mouthon, L. \& Kaveri, S. V. Intravenous immunoglobulin expands regulatory $\mathrm{T}$ cells in autoimmune rheumatic disease. J. Rheumatol. 39, 450-451 (2012).

74. Lutz, H. U. et al. Intravenously applied lgG stimulates complement attenuation in a complement-dependent autoimmune disease at the amplifying C3 convertase level. Blood 103, 465-472 (2004).

75. Sharma, M. et al. Intravenous immunoglobulin-induced IL-33 is insufficient to mediate basophil expansion in autoimmune patients. Sci. Rep. 4, 5672 (2014).

76. Raju, R. \& Dalakas, M. C. Gene expression profile in the muscles of patients with inflammatory myopathies: effect of therapy with IVlg and biological validation of clinically relevant genes. Brain 128, 1887-1896 (2005).

77. Ching, J. K. et al. mTOR dysfunction contributes to vacuolar pathology and weakness in valosin-containing protein associated inclusion body myopathy. Hum. Mol. Genet. 22, 1167-1179 (2013).

78. Ramachandran, N. et al. VMA21 deficiency prevents vacuolar ATPase assembly and causes autophagic vacuolar myopathy. Acta Neuropathol. 125, 439-457 (2013).

79. Mukherjee, S., Karnam, A., Das, M., Babu, S. P. S. \& Bayry, J. Wuchereria bancrofti filaria activates human dendritic cells and polarizes T helper 1 and regulatory T cells via toll-like receptor 4. Commun. Biol. 2, 169 (2019).

80. Chalumeau, C. et al. Kidney cortex cells derived from SV40 transgenic mice retain intrinsic properties of polarized proximal tubule cells. Kidney Int. $\mathbf{5 6}$ 559-570 (1999). 